\title{
Editorial
}

\section{Advances in Applications of Polymer Nanocomposites}

\author{
Yahya A. Ismail (D), Mohammad Luqman, ${ }^{1}$ Rajaram S. Mane $\mathbb{D}^{3,4}{ }^{3,4}$ Yasir E. Greish, \\ and Habib M. Pathan ${ }^{6}$ \\ ${ }^{1}$ Department of Chemistry, University of Calicut, Kerala 673635, India \\ ${ }^{2}$ Chemical Engineering Department, College of Engineering, Taibah University, Yanbu Albahr 41911, Saudi Arabia \\ ${ }^{3}$ Global Frontier R\&D Center for Hybrid Interface Materials, Pusan National University, Busan 609735, Republic of Korea \\ ${ }^{4}$ School of Physical Sciences, SRTM University, Nanded 431-606, India \\ ${ }^{5}$ Department of Chemistry, UAE University, Al Ain, UAE \\ ${ }^{6}$ Advanced Physics Laboratory, Department of Physics, Savitribai Phule Pune University, Pune-07, India
}

Correspondence should be addressed to Yahya A. Ismail; aiyahya123@gmail.com

Received 24 March 2019; Accepted 25 March 2019; Published 22 May 2019

Copyright (C) 2019 Yahya A. Ismail et al. This is an open access article distributed under the Creative Commons Attribution License, which permits unrestricted use, distribution, and reproduction in any medium, provided the original work is properly cited.

Polymers are increasingly being used in numerous cuttingedge technologies owing to their robust behavior, versatile properties, and comparably easier process ability. They serve as excellent host matrices for nanocomposite materials and can easily be tailored to suit various requirements. Polymer nanocomposites involve fillers in the nanometer scale and demonstrate significantly different properties than in bulk due to the variations in the interaction with the host matrices and morphology and created great interest both in the academia and industry. A general feature of most of the polymers, that is, relatively low strength, coupled with low impact strength, has considerably been improved with the emergence of polymer nanocomposites. In this edition, our focus was to address the advances in the applications of polymer nanocomposites. We invited a few publications in the form of original research articles addressing recent advancements in the field of polymer nanocomposites.

The first paper of this issue presents a novel study on preparation, physicochemical characterization, and microrobotics applications of polyvinyl chloride- (PVC-) based polyaniline (PANI)/poly(3,4-ethylene dioxythiophene) (PEDOT): polystyrene sulfonate (PSS)/zirconium(IV) phosphate $(\mathrm{ZrP})$ composite cation exchange ionomeric membranes. The results demonstrated that the developed ionomeric membrane could produce good tip blocking force and displacement. The proposed ionomeric membrane was found suitable for bending movement actuator that may lead it promising for smaller-scale mechanical applications.
The second paper deals with effects of plasticizer (e.g., polydimethylsiloxanol) and modified nanosilica having amine functional groups on the morphology, thermal, mechanical, and rheological properties of biodegradable maleated polylactide (MPLA)/thermoplastic starch (TPS)/nanosilica composites. It was observed that polydimethylsiloxanol helped in enhancing the ductility and the initial thermal stability of the plasticized blend. Nucleation ability and reinforcing effect of maleated $\mathrm{SiO}_{2}$ on plasticized blend crystallization were much better than those of neat $\mathrm{SiO}_{2}$. Neat silica practically had no effect on the thermo-oxidative degradation.

Studies on the miscibility and phase behaviour of polypyrrole (PPy) and polyaniline (PANI) polymer blends are presented in the third paper. The PPy/PANI blends were successfully formed in the DMSO solvent. The polymer blends showed almost amorphous nature in XRD spectra owing to a good intermolecular interaction between PPy and PANI macromolecules, which was confirmed by FT-IR data. The DSC results, especially, for the PPY: PANI $=50: 50 \mathrm{wt} . \%$ blend showed only one glass transition temperature $(\mathrm{Tg})$, indicating that the two polymers are well miscible without undergoing any phase separation.

The fourth paper addresses a novel and facile approach, nanoclay aerogel masterbatch, toward making exfoliated polymer [polyethylene glycol (PEG)]-clay nanocomposites through a melt-mixing process to modify the clay layer through PEG-clay intercalation and PEG-hydrogen bonding. 
This state was maintained with a small amount of the anionic polymer hydrogel, e.g., kappa-carrageenan (KC), and turning it into a highly porous and fragile structure by freeze-drying, thus a so-called nanoclay aerogel masterbatch. The highest nanoclay loading capacity (67 wt.\%) of the achieved nanoclay aerogel masterbatch was selected to prepare PS-clay nanocomposites via a melt-mixing process. The outstanding transparency and brightness were remarked from the specimens prepared by using the nanoclay aerogel masterbatch. The brownish specimens were observed by using organoclay. The significant improvements of thermal and mechanical properties were observed from the nanocomposites prepared using the nanoclay aerogel masterbatch.

The fifth paper of this special issue sheds light on dielectric properties of $\mathrm{TiO}_{2} /$ room temperature vulcanized (RTV) silicone rubber micro- and nanocomposites for highvoltage electrical insulation. The surfactant Triton X-100 greatly improves the dispersal of micro- and nanoparticles across the surface to produce more homogeneous composites that have improved dielectric properties. The influence of the filler concentration and particle size on the dielectric behaviour of the nanocomposites and the microcomposite was also studied. There was an improvement in the permittivity values for the microcomposites as compared to those of nanocomposites for all frequencies.

\section{Conflicts of Interest}

The editors declare that there are no conflicts of interest.

Yahya A. Ismail

Mohammad Luqman

Rajaram S. Mane

Yasir E. Greish

Habib M. Pathan 


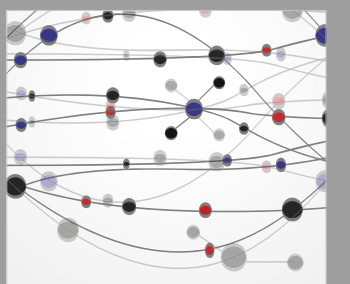

The Scientific World Journal
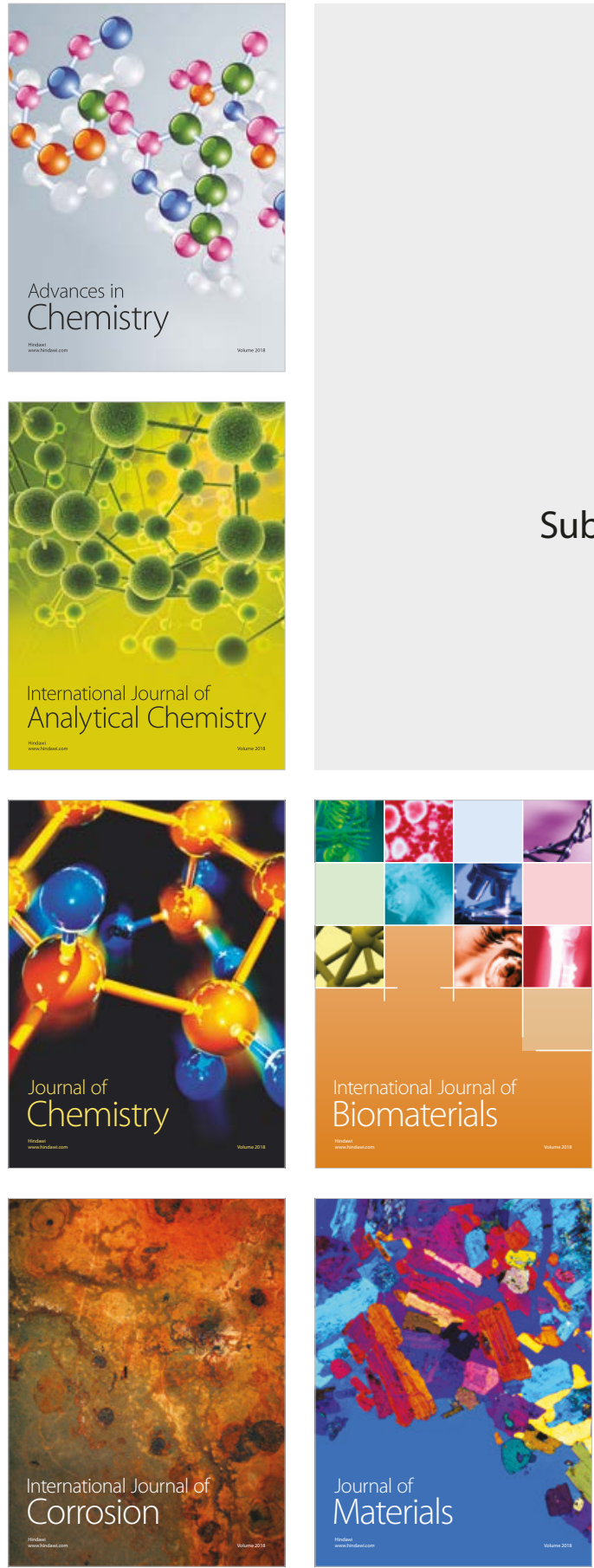

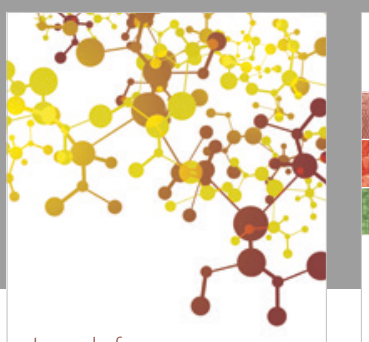

Journal of

Applied Chemistry
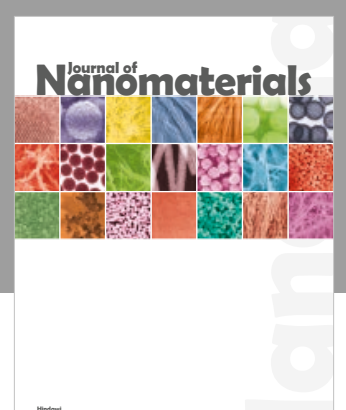

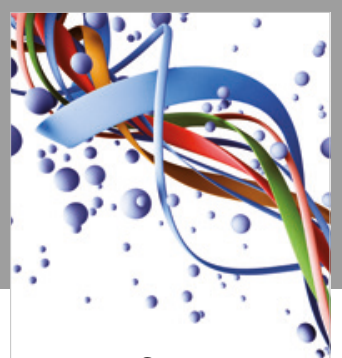

Scientifica

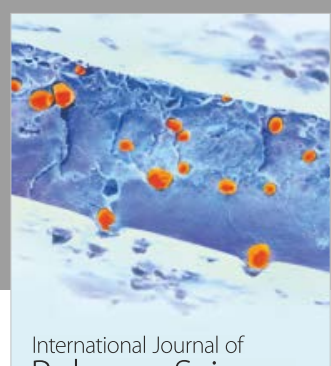

Polymer Science

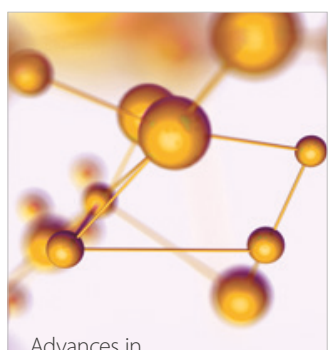

Physical Chemistry
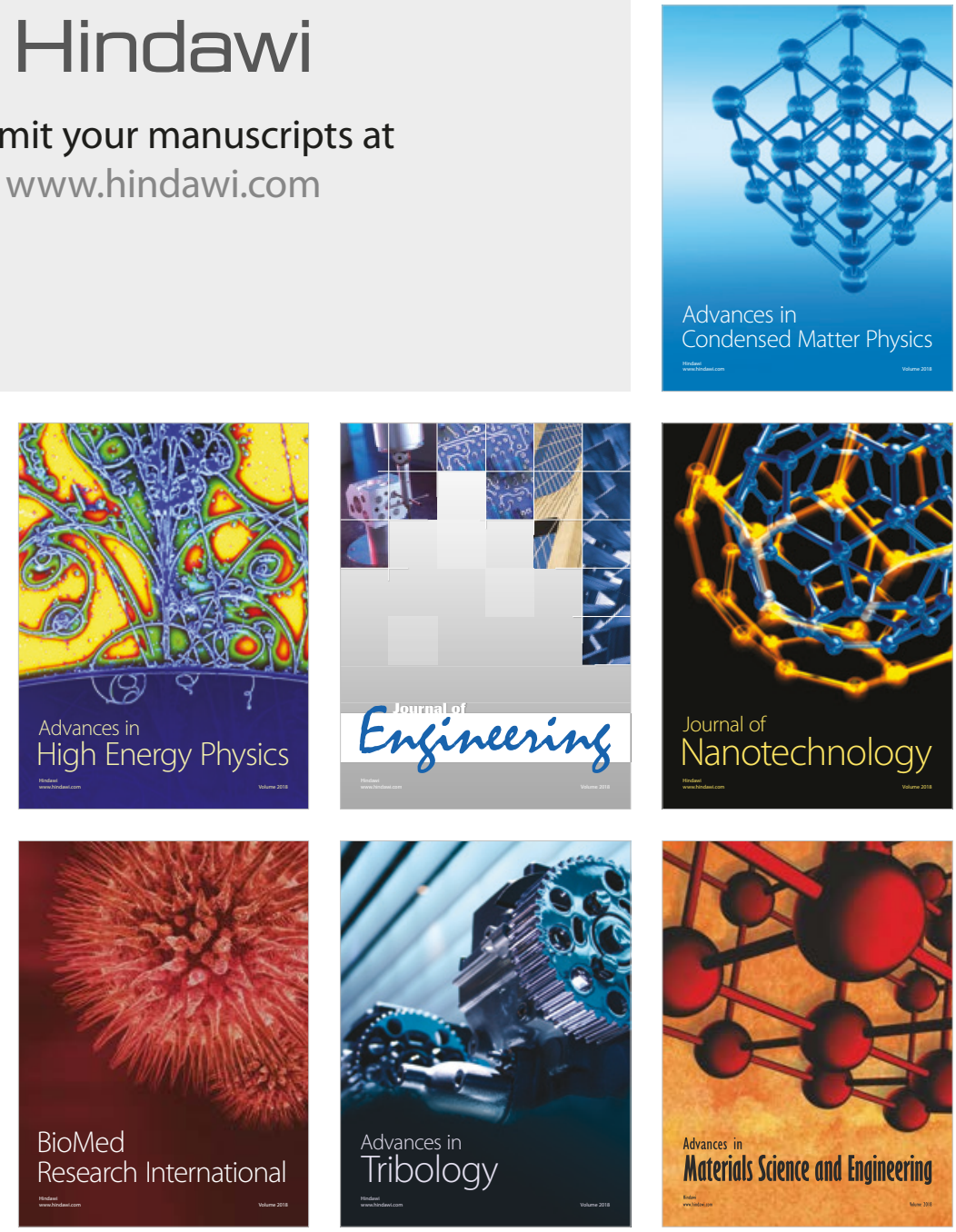